\title{
Alongamento passivo agudo não afeta a atividade muscular máxima dos ísquiotibiais
}

\author{
The acute passive stretching does not affect the maximal muscular \\ activity of the hamstrings
}

\author{
A.S. Bley, P.S. Nardi, P.H. Marchetti
}

ARTIGO ORIGINAL | ORIGINAL ARTICLE

O alongamento agudo pode influenciar a produção de força em diferentes tarefas e performances esportivas. O objetivo do presente estudo foi investigar a influência do alongamento passivo agudo na atividade elétrica do músculo bíceps femoral (BF) e na força dos músculos isquiotibiais (IT). Participaram 14 adultos jovens, sedentários fizeram parte deste estudo. Foram requisitados aos sujeitos que realizassem contrações isométricas máximas antes e após um protocolo de alongamento passivo. O protocolo de alongamento foi composto de 3 tentativas de 60 segundos e repouso de 15 segundos entre cada tentativa. Os sujeitos foram submetidos a aquisições eletromiográficas do músculo BF e da força máxima dos IT, em pré e pós-alongamento. Foram analisadas a força isométrica máxima (FIM), atividade eletromiográfica integrada (IEMG) e frequência mediana (FMed) do sinal EMG em ambas as condições, através de um teste $T$ de student pareado. A FIM $(14.1 \pm 4.9$ e $13.1 \pm 4.5 \mathrm{Kgf}$, respectivamente, $p=$ .12), a IEMG (208.4 \pm 89.9 e $189.3 \pm 75$ V.s, respectivamente, $p=.059)$, e a FMed $(22.7 \pm 4$ e 23.4 $\pm 5.5 \mathrm{~Hz}$, respectivamente, $p=.52$ ) do músculo BF não apresentaram diferenças significativas entre as condições de pré e pós-alongamento. Os efeitos agudos do alongamento passivo não influenciaram o padrão de ativação elétrica do BF ou a força dos IT.

Palavras-chave: isquiotibiais, alongamento passivo, eletromiografia, força

ABSTRACT

The acute stretching may influence the force production in different tasks and sports performances. The aim of this study was to investigate the influence of acute passive stretch on the electrical activity of the biceps femoris (BF) and hamstring muscle (HS) strength. Fourteen young adults, sedentary took part of this study. The subjects were asked to produce the maximal isometric contractions before and after a passive stretching protocol. The stretching protocol consisted of three attempts at 60 seconds and resting 15 seconds between each attempt. The subjects were analyzed using the following techniques: maximal isometric force (MIF), integrated electromyography (IEMG) and median frequency (Fmed) of the EMG signal of the BF before and after stretching. We performed the MIF, IEMG and Fmed of the EMG signal under these conditions and compared using a paired Student $t$ test. The MIF of the biceps femoris $(14.1 \pm 4.9$ e $13.1 \pm 4.5 \mathrm{Kgf}$, respectively, $p=.12)$, IEMG $(208.4 \pm 89.9$ e $189.3 \pm$ 75 V.s, respectively, $p=.059)$, and FMed $(22.7 \pm 4$ e $23.4 \pm 5.5 \mathrm{~Hz}$, respectively, $p=.52)$ showed no significant differences between conditions before and after stretching. The acute effects of passive stretching did not significantly influence the pattern of electrical activation of the BF and the maximal isometric force produced by the HS.

Keywords: hamstrings, passive stretching, electromyography, strength

Submetido: 01.02.2012 | Aceite: 02.12.2012

P. H. Marchetti. Grupo de Pesquisa em Neuromecânica do Treinamento de Força (GNTF), Faculdade de Educação Física de Sorocaba (ACM), Sorocaba, Brasil.

A. S. Bley. Cursos de Fisioterapia, UNINOVE, São Paulo, Brasil.

P. S.M. Nardi. Curso de Fisioterapia, UNIP, Sorocaba, Brasil.

Endereço para correspondência: Paulo H. Marchetti, Universidade Metodista de Piracicaba, Grupo de Pesquisa em Performance Humana, Rodovia do Açúcar Km 156, Bloco 7, Sala 32, Taquaral, 13400-911 - Piracicaba, SP, Brasil.

E-mail: dr.pmarchetti@gmail.com 
O alongamento é uma técnica utilizada para aumentar a flexibilidade e ganhar amplitude de movimento, essas alterações ajudam na prevenção de lesões músculo-esqueléticas, aumentam o desempenho nas atividades e permite que as articulações realizem o arco de movimento normal (Bandy \& Sanders, 2003). Existem três tipos de exercícios de alongamento para aumento da flexibilidade: alongamento balístico, estático e facilitação proprioceptiva neuromuscular (FNP). O alongamento estático, também chamado de passivo, é um método pelo qual o músculo é estirado lentamente, até obter uma leve tensão (confortável e sem dor), onde esta posição é mantida por alguns segundos. $\mathrm{O}$ alongamento deve ser lento e prolongado com o intuito de evitar a estimulação do fuso neuromuscular evitando, desta forma, a contração reflexa, e, além disso, exercer tensão sobre o Órgão Tendinoso de Golgi que produz estímulo inibitório à contração do músculo alongado (Bandy \& Sanders, 2003; Fleck \& Kraemer, 1999).

A compreensão dos efeitos de uma sessão aguda de alongamento em outras capacidades físicas (ex. Força) é fundamental para a adequação da prescrição do treinamento com sessões complexas, assim como em programas de reabilitação, prevenindo possíveis efeitos negativos na capacidade subsequente. $\mathrm{O}$ alongamento agudo pode influenciar a produção de força em diferentes tarefas e performances esportivas. Este fenômeno é chamado de déficit de força induzido pelo alongamento (DFIA) (Herda, Cramer, Ryan, \& McHugh, 2008; Ryan et al., 2008). O DFIA pode estar relacionado à dose-resposta do treinamento, tipo de alongamento (estático, dinâmico ou FNP) (Herda, et al., 2008) ou características musculares específicas (tipos de fibras), podendo durar entre 6 a 90 minutos (Ryan, et al., 2008). Existem duas hipóteses primárias para explicar tal fenômeno: (1) Fatores neurais como a diminuição da ativação muscular, alteração das estratégias de controle motor, efeito de inibição do sistema nervoso central e diminuição de sensitividade dos proprioceptores articulares e musculares (Herda, et al., 2008); (2) Fatores mecânicos como a diminuição da rigidez músculo-tendínea que pode afetar a curva de comprimento-tensão e/ou a taxa da velocidade de encurtamento dos sarcômeros (Cramer \& Housh, 2005; Fowles, Sale, \& MacDougall, 2000; Herda, et al., 2008; Ryan, et al., 2008).

Diversos estudos têm reportado que o alongamento antes do exercício ou performance esportiva reduz a força isométrica ou dinâmica, a atividade eletromiográfica (EMG) ou mecanomiográfica (MMG), o pico de torque concêntrico, além de reduzir a altura do salto, taxa de desenvolvimento de força e pico de sprint em atletas (Herda, et al., 2008; La Torre et al., 2010; Turki et al., 2011). Entretanto, alguns estudos não verificaram tais efeitos na redução da força, EMG ou MMG (Beedle, Rytter, Healy, \& Ward, 2008; Brentano, Rodrigues, \& Martins Kruel, 2008).

Portanto, o objetivo do presente estudo foi analisar o efeito agudo de uma sessão de alongamento passivo na atividade elétrica do músculo bíceps femoral e na produção de força do grupo de músculos isquiotibiais. A hipótese principal do estudo é que o alongamento passivo agudo reduz a força isométrica máxima dos isquiotibiais e a ativação muscular produzida pelo bíceps femoral.

\section{MÉTODO}

\section{Amostra}

Participaram do estudo 14 adultos jovens sedentários, com média ( \pm desvio padrão) de idade de $25 \pm 3$ anos. Os critérios de inclusão adotados foram indivíduos sem qualquer prática de atividade física por no mínimo 2 anos, sem disfunções na locomoção, ausência de patologias prévias no membro inferior dominante ou diminuição na flexibilidade dos músculos ísquiotibiais (confirmado quando não houvesse extensão total do joelho com o quadril flexionado em $90^{\circ}$ ). A metodologia 
proposta foi formulada respeitando resoluções 196/96 do Conselho Nacional de Saúde e foi aprovada pelo Comitê de Ética em Pesquisa da Universidade Nove de Julho - UNINOVE (parecer $\mathrm{n}^{\circ}$ 341553). Todos os participantes foram esclarecidos quanto à metodologia utilizada e assinaram um Termo de Consentimento Livre e Esclarecido.

\section{Procedimentos}

Inicialmente, os voluntários foram submetidos à coleta do sinal eletromiográfico (EMG) do músculo bíceps femoral (BF) (músculo integrante dos isquiotibiais (IT)), em decúbito ventral, com o joelho flexionado em $80^{\circ}$ do membro dominante, e tornozelo preso à uma corrente/célula de carga. Os sujeitos realizaram um breve aquecimento localizado e familiarização, realizando a tarefa de flexão do joelho isométricamente durante 5 segundos por 2 séries intervaladas por 1 minuto de descanso. Então, os sujeitos foram instruídos a realizarem a máxima força possível contra uma resistência externa fixa (corrente/célula de carga), resistindo isometricamente à flexão de joelho, utilizando o membro dominante (definido pelo membro preferido para chutar uma bola (Maulder \& Cronin, 2005)). Foram realizadas quatro contrações isométricas voluntárias máximas (CIVM) dos IT, sendo três antes do protocolo de alongamento (condição pré-alongamento) e uma após o protocolo de alongamento (condição pós-alongamento). As condições pré-alongamento foram intercaladas por 1 minuto de intervalo. Após a aquisição do sinal EMG e da força máxima produzida, os participantes foram submetidos a uma sessão de alongamento passivo para o IT, em decúbito dorsal, onde o avaliador manteve o joelho em extensão máxima e lenta e passivamente realizou a flexão de quadril até o voluntário sentir uma tensão confortável na região posterior da coxa. O protocolo de alongamento foi realizado através de 3 tentativas de 60 segundos e repouso de 15 segundos entre cada tentativa, totalizando
180 segundo de estímulo. Imediatamente após o alongamento os mesmos procedimentos da coleta dos dados iniciais foram realizados novamente para fins de comparação.

Para a coleta dos dados, foi utilizado um eletromiógrafo de 16 canais (EMG System do Brasil), com filtro de banda de frequência 10 $500 \mathrm{~Hz}$, amplificador com ganho de 100x (total final de 1000 x) e célula de carga (marca) presa a uma corrente e fixa ao tornozelo do sujeito. Um par de eletrodos de superfície, circulares, auto-adesivos, $\mathrm{Ag} / \mathrm{AgCl}$, do tipo ativo, bipolar, distância entre eletrodos de $2 \mathrm{~cm}$, diferencial e razão do modo comum de rejeição igual a $-80 \mathrm{~dB}$ (auto-adesivo e descartável da marca Meditrace), foi colocado sobre o BF, segundo as recomendações do SENIAM (Surface EMG for à non-invasive assessment of muscles) (Hermens, Freriks, Disselhorst-Klug, \& Rau, 2000). Prévia a colocação dos eletrodos foi realizada tricotomia e limpeza do local. O eletrodo de referência foi colocado no maléolo lateral do tornozelo. A aquisição dos dados foi feita a uma frequência de $1000 \mathrm{~Hz}$ e os dados foram processados através de uma rotina escrita no software Matlab (Mathworks Inc., EUA).

A aquisição e análise temporal e de frequências da atividade elétrica do músculo BF seguiu as recomendações da Sociedade Internacional de Eletrofisiologia Cinesiológica (ISEK). Todos os sinais EMGs foram tratados para posterior comparação e análise conforme preconizado por Winter (1990). Foram removidos o primeiro e último segundo do sinal EMG visando evitar ajustes corporais ou o efeito da fadiga. Para a análise temporal e espectral do sinal EMG foi utilizado o primeiro segundo de aquisição, após a estabilização da força (avaliada através da célula de carga), evitando assim, o efeito da fadiga neuromuscular. $\mathrm{O}$ processamento do sinal EMG seguiu a seguinte ordem (Marchetti \& Duarte, 2011): os sinais EMG foram filtrados com um filtro de $4^{\text {a }}$ ordem, passa banda entre $20-400 \mathrm{~Hz}$, e atraso de fase zero. Foi utilizada a root-mean square (RMS) com uma janela de 
$100 \mathrm{~ms}$, para a amplitude do sinal EMG (RMS EMG), e então o sinal RMS EMG foi integrado (IEMG). Para a análise no domínio de frequências, foi utilizada a transformada rápida de Fourier (FFT) para o intervalo de 1 segundo. A frequência mediana (FMed) do espectro de potência foi calculada para cada condição e utilizada para posterior análise. Para a condição pré-alongamento a média dos valores foi utilizada para posterior análise. O coeficiente de correlação interclasses (Hewett, Linderfeld, Riccobene, \& Noyes) das 3 tentativas na condição pré-alongamento foi de FIM (.94); IEMG (.85) e FMed (.79).

\section{Análise Estatística}

A análise estatística descritiva envolveu medidas de tendência central e dispersão. Todos os dados foram reportados através da média e desvio padrão (DP) da média. A normalidade e homogeniedade das variâncias foram verificadas utilizando o teste de Kolmogorov-Smirnov e de Levene, respectivamente. Teste $\mathrm{t}$ de student pareado foi utilizado para verificar as diferenças entre as condições de pré e pós-alongamento para os valores de IEMG do músculo BF, pico de força e frequência mediana. Um nível de significância ( $\alpha$ ) de .05 foi utilizado para todos os testes estatísticos, através do software SPSS versão 18.0.

\section{RESULTADOS}

Não foram observadas diferenças significativas entre as condições pré-alongamento e pós-alongamento, para as variáveis analisadas: força máxima $(p=.12)$, IEMG $(p=.059) \mathrm{e}$ FMed $(p=.52)$, como mostra a Figura 1 .

\section{DISCUSSÃO}

O objetivo do presente estudo foi analisar o efeito agudo de uma sessão de alongamento passivo na atividade elétrica do músculo $\mathrm{BF}$ e na produção de força do grupo de músculos IT. Os dados do presente estudo não corroboram com a hipótese principal, de que o alongamento

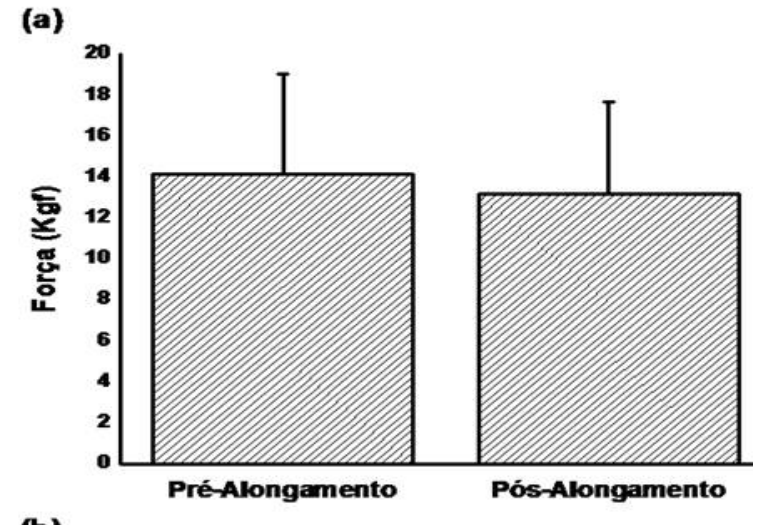

(b)

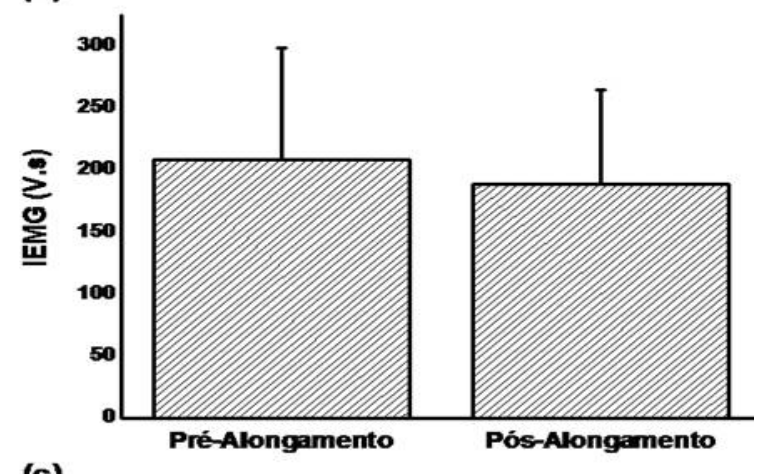

(c)

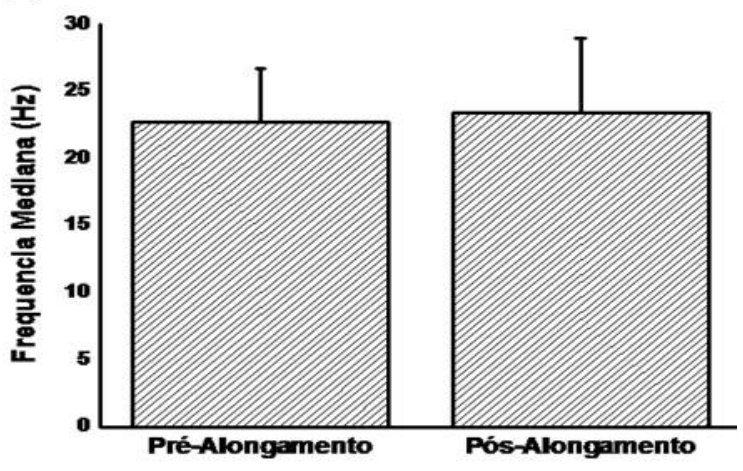

Figura 1. Média e desvio padrão da (a) força isométrica máxima dos isquiotibiais, (b) atividade eletromiográfica integrada (IEMG) e (c) frequência mediana do músculo bíceps femoral, nas condições pré e pós-alongamento.

passivo agudo reduz a força isométrica máxima dos IT e a ativação muscular produzida pelo BF.

Diversos estudos têm demonstrado que as práticas do alongamento, antes de atividades que exijam força e potência musculares, podem levar a queda aguda da força máxima e da potência dos grupos musculares previamente alongados (Behm, Bambury, Cahill, \& Power, 2004; Kokkonen, Nelson, \& Cornwell, 1998; Marek et al., 2005; Zakas, Doganis, Papakonstadinov, Sentelidis, \& Vamvakoudise, 2006). 
Na revisão de literatura realizada por Rubini, Costa e Gomes (2007) foi verificado que de 27 pesquisas visando estudar o efeito agudo do alongamento passivo, 21 apresentaram déficits significativos de força muscular e apenas 6 não encontraram diminuição na força muscular. Brentano et al. (2008) citam que diferentes técnicas de aquecimento são realizadas pré-participação em atividades físicas, dentre elas destaca-se o alongamento passivo. Seu estudo afirma que a aplicação do alongamento antes do exercício não prejudica o desempenho da força muscular.

O presente estudo não verificou diferenças significativas na produção de força máxima para as condições de pré e pós-alongamento, corroborando o estudo de Beedle et al. (2008). O tipo de protocolo ou o grau de tensão produzido no músculo durante o alongamento poderiam ser fatores influenciadores dos resultados (Garcia-López et al., 2010; La Torre, et al., 2010; Ryan, et al., 2008; Sheard \& Paine, 2010), produzindo diferentes modificações nos componentes elásticos em série ou mesmo no controle neural dos sujeitos avaliados (Beedle, et al., 2008; Molacek, Conley, Evetovich, \& Hinnerichs, 2010). Outro possível fator que poderia ter influenciado os resultados apresentados seria o posicionamento articular realizado durante o protocolo de alongamento, pois estudos mostram que a queda de força pós-alongamento é ângulo-específico (La Torre, et al., 2010; Nelson, Allen, Cornwell, \& Kokkonen, 2001). O presente estudo utilizou 90 graus de flexão de joelhos para avaliar a força máxima isométrica, entretanto o estudo de Nelson et al.(2001) mostrou que o efeito de queda de força foi mais evidente em ângulos articulares próximos a máxima extensão do joelhos.

Quanto aos efeitos do alongamento agudo nas alterações no padrão mioelétrico, alguns estudos observaram queda de força muscular, mas sem alterações no sinal EMG ou mecanomiográfico dos músculos analisados, propondo alterações apenas na unidade músculo-tendínea
(Beedle, et al., 2008), não corroborando o presente estudo. Entretanto, nenhum estudo analisou os efeitos do alongamento agudo nas alterações espectrais do sinal EMG. As modificações no conteúdo de frequências do sinal EMG podem contribuir no entendimento do controle neuromuscular sob o efeito agudo do alongamento. Desta forma, considerando as diferentes análises realizadas (temporal e espectral) do sinal EMG, se pode considerar que não houve grandes alterações no controle de movimento durante as condições analisadas, assim o alongamento perece não ter tido efeito na inibição da ação muscular.

As implicações clínicas deste estudo, diante de um programa de reabilitação baseado no fortalecimento muscular, mostram que o alongamento prévio não parece ter influência negativa na busca do reequilíbrio muscular para estabilização articular.

\section{CONCLUSÕES}

Concluiu-se que os efeitos agudos do alongamento passivo não influenciaram significativamente o padrão de ativação muscular do bíceps femoral ou a produção máxima de força isométrica dos isquiotibiais.

\section{Agradecimentos: \\ Nada declarado.}

\section{Conflito de Interesses:}

Nada declarado.

\section{Financiamento:}

Nada declarado.

\section{REFERÊNCIAS}

Bandy, W. D., \& Sanders, B. (2003). Exercício terapêutico, técnicas para intervenção. Rio de janeiro: Ed. Guanabara Koogan.

Beedle, B., Rytter, S. J., Healy, R. C., \& Ward, T. R. (2008). Pretesting static and dynamic stretching does not affect maximal strength. Journal of strength and conditioning research, 22(6), 1838- 
1843. doi: 10.1519/JSC.0b013e31817b0433

Behm, D. G., Bambury, A., Cahill, F., \& Power, K. (2004). Effect of acute static stretching on force, balance, reaction time, and movement time. Medicine Science and Sports Exercise 36(8), 1397-1402. doi: 10.1249/01

Brentano, M. A., Rodrigues, L. P., \& Martins Kruel, L. F. (2008). Efeitos de diferentes sessões de aquecimento no torque e amplitude articular de homens jovens. Revista Brasileira de Educação Física e Esporte, 22(11), 53-62. doi: http:// www.revistasusp.sibi.usp.br/pdf/rbefe/v22n1/ v22n1a5.pdf.

Cramer, J. T., \& Housh, T. J. (2005). The acute effects of static stretching on peak torque, mean power output, electromyography, and mechanography. Journal of Sports Physiology, 25(6), 530-539. doi: digitalcommons.unl.edu/dissertations/ AAI3092535

Fleck, S. J., \& Kraemer, W. J. (1999). Fundamentos do treinamento de força muscular (2 ed.). São Paulo: Manole.

Fowles, J. R., Sale, D. G., \& MacDougall, J. D. (2000). Reduced strength after passive stretch of the human plantarflexors. Journal of Applied Physiology, 89, 1179-1188. doi: www.ncbi.nlm. nih.gov/pubmed/10956367.

Garcia-López, D., Izquierdo, M., Rodriguez, S., González-Calvo, G., Sainz, P., Abadía, O., \& Herrero, A. J. (2010). Interset stretching does not influence the kinematic profile of consecutive bench-press sets. Journal of strength and conditioning research., 24(5), 1361-1368. doi: 10.1519/JSC.0b013e3181cf780d

Herda, T. J., Cramer, J. T., Ryan, E. D., \& McHugh, M. P. (2008). Acute effects of static versus dynamic stretching on isometric peak torque, electromyography, and mechanography of the biceps femoris muscle. Journal of strength and conditioning research, 22(3), 809-817. doi: 10.1007/s00421-004-1199-X

Hermens, H. J., Freriks, B., Disselhorst-Klug, C., \& Rau, G. (2000). Development of recommendations for SEMG sensors and sensor placement procedures. Journal of Electromyography and Kyne- siology., 10, 361-374.

Hewett, T. E., Linderfeld, T. N., Riccobene, J. V., \& Noyes, F. R. (1999). The effect of neuromuscular training on the incidence of knee injury in female athletes: A prospective study. American Journal of Sports Medicine., 27, 699-706.

Kokkonen, J., Nelson, A. G., \& Cornwell, A. (1998). Acute muscle stretching inhibits maximal strength perfomance. Research of Quarterly Exercise and Sport 69(4), 411-415. doi: 10.1055/s2002-19271

La Torre, A., Castagna, C., Gervasoni, E., Ce, E., Rampichini, S., Ferrarin, M., \& Merati, G. (2010). Acute effects of static stretching on squat jump performance at different knee starting angles. Journal of strength and conditioning research, 24(3), 687-694. doi: 10.1519/ JSC.0b013e3181c7b443

Marchetti, P. H., \& Duarte, M. (2011). Eletromiografia: uma breve revisão sobre os procedimentos de aquisição do sinal. Terapia Manual. doi: www.revistatm.com.br/index.php/revista/ article.

Marek, S. M., Cramer, J. F., Fincher, A. L., Massey, L. L., Dangelmaier, S. M., \& Purkayastha, S. (2005). Acute effects of static and proprioceptive neuromuscular facilitation stretching on muscle strenght and power output. Journal of Athletic Training, 40(2), 94-103. doi: 10.3900/ fpj.8.4.264

Maulder, P., \& Cronin, J. (2005). Horizontal and vertical jump assessment: reliability, symmetry, discriminative and predictive ability. Physical Therapy in Sport, 6(2), 74-82. doi: http://dx.doi. org/10.1016/j.ptsp.2005.01.001.

Molacek, Z. D., Conley, D. S., Evetovich, T. K., \& Hinnerichs, K. R. (2010). Effects of low- and high volume stretching on bench press performance in colegiate football players. Journal of strength and conditioning research., 24(3), 711-716. doi: 10.1519/JSC.0b013e3181c7c242

Nelson, A. G., Allen, J. D., Cornwell, A., \& Kokkonen, J. (2001). Inhibition of maximal voluntary isometric torque production by acute stretching is joint-angle specific. 
86 | A.S. Bley, P.S. Nardi, P.H. Marchetti

Research Quartely Exercise Sport, 72, 68-70. doi: 10.1080/02640410410001730205

Rubini, E. C., Costa, A. L. L., \& Gomes, P. S. C. (2007). The effects of stretching on strength performance. Sports Medicine, 37, 213-224.

Ryan, E. D., Beck, T. W., Herda, T. J., Hull, H. R., Hartman, M. J., Stout, J. R., \& Cramer, J. T. (2008). Do practical durations of stretching alter muscle strength? A dose-response study. Medicine and Science of Sports Exercise, 40(8), 15291537. doi: 10.1519/JSC.0b013e31815ef202

Sheard, P. W., \& Paine, T. J. (2010). Optimal contraction intensity during proprioceptive neuromuscular facilitation for maximal increase of range of motion. Journal of strength and conditioning research., 24(2), 416-421. doi: 10.1519/ JSC.0b013e3181c50a0d
Turki, O., Chaouachi, A., Drinkwater, E. J., Chtara, M., Chamari, K., Amri, M., \& Behm, D. G. (2011). Ten minutes of dynamic stretching is sufficient to potentiate vertical jump performance characteristics. Journal of strength and conditioning research., 25(9), 2453-2463. doi: 10.1519/JSC.0b013e3181d0b109

Winter, D. A. (1990). Biomechanics and motor control of human movement. USA.

Zakas, A., Doganis, G., Papakonstadinov, V., Sentelidis, T., \& Vamvakoudise, E. (2006). Acute effects os static stretching duration isokinetic peak torque production of soccer players. Journal of Bodywork and Moviment Theraphy. , 10, 89-95. doi: 10.1016/j.jbmt.2005.04.007 